\title{
FLUX DISTRIBUTION OF SOLAR INTRANETWORK MAGNETIC FIELDS
}

\author{
JINGXIU WANG \\ Beijing Astronomical Observatory, Chinese Academy of Sciences, Beijing 100080, China \\ and
}

HAIMIN WANG, FRANCES TANG, JEONGWOO W. LEE and HAROLD ZIRIN

Big Bear Solar Observatory, California Institute of Technology, Pasadena, CA 91125, U.S.A.

(Received 5 December, 1994; in revised form 5 May, 1995)

\begin{abstract}
Big Bear deep magnetograms of June 4, 1992 provide unprecedented observations for direct measurements of solar intranetwork (IN) magnetic fields. More than 2500 individual IN elements and 500 network elements are identified and their magnetic flux measured in a quiet region of $300 \times 235$ arc sec. The analysis reveals the following results:

(1) IN element flux ranges from $10^{16} \mathrm{Mx}$ (detection limit) to $2 \times 10^{18} \mathrm{Mx}$, with a peak flux distribution of $6 \times 10^{16} \mathrm{Mx}$.

(2) More than $20 \%$ of the total flux in this quiet region is in the form of IN elements at any given time.

(3) Most IN elements appear as a cluster of mixed polarities from an emergence center (or centers) somewhere within the network interior.

(4) The IN flux is smaller than the network flux by more than an order of magnitude. It has a uniform spatial distribution with equal amount of both polarities.

It is speculated that IN fields are intrinsically different from network fields and may be generated from a different source as well.
\end{abstract}

\section{Introduction}

The intranetwork (IN) magnetic fields are at the tail end of the spectrum of solar magnetic fields in the photosphere. They are weakest in field strength and smallest in flux. They appear as small elements of magnetic fields inside the network (supergranular) cells. Like the network fields, their presence does not seem to be dependent on the latitude on the globe. Unlike network fields, however, the IN fields are not affected by decaying active region fields.

IN fields were first observed by Livingston and Harvey (1975) and Smithson (1975). They were described as 'discrete elements' of mixed polarities 'interior to the network'. In recent years progress was made in the understanding of IN's morphology, dynamics and some quantitative aspects from time sequences of deep magnetograms obtained at the Big Bear and Huairou Solar Observatories (Livi, Wang, and Martin, 1985; Martin, 1984, 1988, 1990; Shi et al., 1990; Wang et al., 1985; Wang, Zirin, and Shi, 1989; Wang and Shi, 1988; Wang and Zirin, 1988; Zirin, 1985, 1987, 1993).

The first Stokes $V$ line ratio measurement made by Keller et al. (1994) has placed an upper limit on the intrinsic strength of IN fields at $1000 \mathrm{G}$ and with $68 \%$ 
probability at $500 \mathrm{G}$. By using an infrared array technique, Lin (1994) was able to directly measure the Zeeman splitting of the Fe I $15648.5 \AA$ and Fe I $15652.9 \AA$ lines of the IN fields. His result revealed that the fields typically have field strengths around $500 \mathrm{G}$.

The current knowledge about IN fields may be summarized as follows: (1) They consist of 'point-like' elements of mixed polarities within the network cells. (2) IN flux elements move at a speed of $0.3-1.0 \mathrm{~km} \mathrm{~s}^{-1}$, but they do not always move radially toward the cell boundaries (Zirin, 1985). (3) They are intrinsically weak, each estimated to have a total flux between $10^{16}$ and $10^{17} \mathrm{Mx}$ (Zirin, 1987). (4) They interact with the network elements upon contact, either 'canceling' with oppositepolarity elements or merging with same-polarity elements (Livi, Wang, and Martin, 1985). (5) Their lifetime has been estimated by several groups, but not statistically determined. A wide range of lifetimes has been reported. High temporal resolution magnetograms show that some of the IN elements are stable for at least several hours.

While most of the network elements can be easily observed, most of the IN fields are difficult to observe because of their smaller size and low flux density. The June 4, 1992 data obtained at Big Bear provided the first set of observation with which a statistical study of the IN and network fields may be carried out.

\section{Data Analysis}

\subsection{DATA}

The data on June 4, 1992 are the best quiet region observations ever obtained at BBSO. The region is near the center of the Sun at S10 W3. The magnetograms were acquired by integrating 4096 video frames, then recording in a 16-bit memory. The calibration was made by the method described by Varsik (1994). The 7-hr observation yielded 73 magnetograms, which were registered by crosscorrelation. The sequence was then made into a movie from which the motion and evolution of the magnetic elements can be traced.

Throughout this paper, the term 'magnetic element' does not imply an elementary flux tube, which is beyond our present knowledge. It merely refers to an observational entity: the intersection of a bundle of magnetic field lines with the photosphere .

Individual IN elements were identified, and their flux measured. Figure 1 is a magnetogram at 16:49 UT when the seeing was the best. The flux measurement is based on this magnetogram. Solar south is at the top, east is to the right. The field of view is $617 \times 473$ pixels. Each pixel is 0.5 arc sec. Enhanced network can be seen in most of the eastern part of the field of view, while quiet network can be seen in parts of the western half.

Since IN fields have low flux density (the magnitude of the vector $\mathbf{B}$ in Maxwell's equations in a medium), noise in the magnetograms could be mis- 


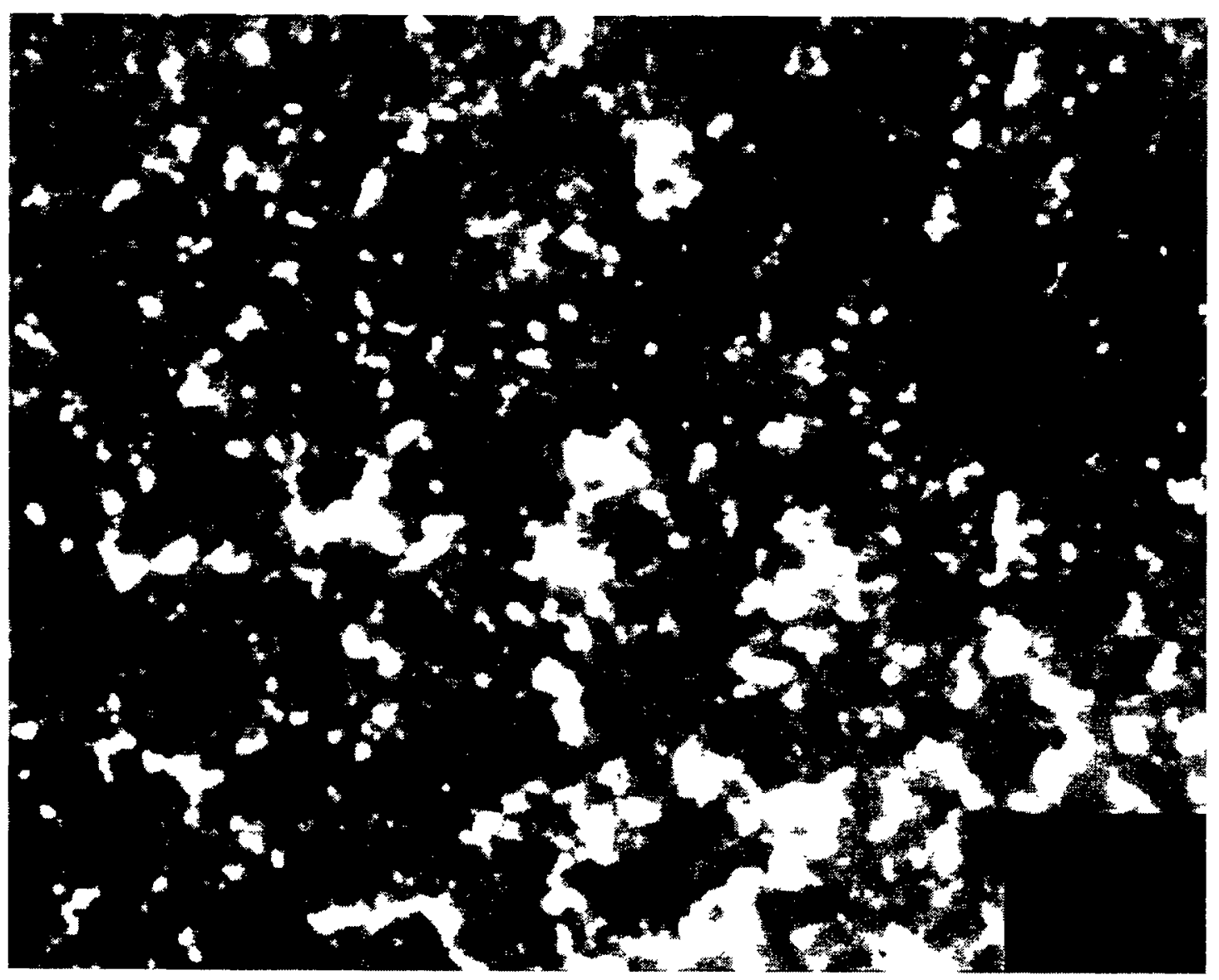

Fig. 1. Magnetogram of a quiet region at 16:49 UT on June 4, 1992. South is top, east is to the right. The field of view is $308 \times 237$ arc sec centered at S10 W3.

construed as IN magnetic elements. To carefully determine the noise level, we select another magnetogram taken 5 min after the best seeing frame (Figure 1), and subtract the two (avoiding the network boundary areas). Since the IN field does not change appreciably in such a short time interval, the subtraction will yield the noise of the magnetograms. The average r.m.s. value for the 20 network cells is $3.0+0.2 \mathrm{G}$, which places an upper limit of $2.1 \mathrm{G}$ to the real noise level in a single magnetogram.

To match the real spatial resolution determined by the atmospheric seeing, a $2 \times 2$ pixel smooth average was made before the flux measurements were made. This further increased the ratio of signal to noise. The final noise level after smoothing is around $1 \mathrm{G}$ for the best magnetograms with a spatial resolution of 2 arc sec.

\subsection{IDENTIFICATION OF IN ELEMENTS}

In most cases, it is not difficult to distinguish the IN magnetic elements from the network elements. Three criteria are used to separate the two. (1) Flux density: our empirical results from magnetograms with spatial resolution of 1-2 arc sec indicate a maximum flux density higher than $40 \mathrm{G}$ for network and lower for IN 
fields. (2) Motion: network elements move at an average speed of $0.06 \mathrm{~km} \mathrm{~s}^{-1}$, while IN elements move at a much higher average speed of $0.4 \mathrm{~km} \mathrm{~s}^{-1}$ (Zirin, 1985). (3) Location: IN elements are within the supergranular network. The latter has a lifetime of 50 to 100 hours (Wang et al., 1989; Liu et al., 1994) and can be easily identified most of the time.

For the study, a magnetic element is defined as a flux patch with a maximum flux density higher than $2 \mathrm{G}$ and an area larger than $1(\operatorname{arcsec})^{2}$. This results in a detection limit for magnetic flux of $10^{16} \mathrm{Mx}$.

The flux measurements for IN and network magnetic elements were made with an interactive data language (IDL) procedure. The elements are identified visually. The boundary of the element is then traced with the mouse and its area, total flux and maximum flux density are measured. Before flux measurements, all pixels with flux density lower than $1 \mathrm{G}$ were set to $0 \mathrm{G}$ (appears as gray in the image), so that the periphery of an element is drawn at the $1 \mathrm{G}$ level. It is assumed that the flux distribution in an element is gaussian with a peak in flux density. Thus, two closely connected elements are separated at the line of lowest flux densities between them. The IN elements usually are well separated in the magnetogram, as is shown in Figure 1. To separate the network elements which often clump together, the dynamic range of the display is raised such that the elements shrink in size and become distinct. For IN flux measurements, the network elements are masked out first by use of the IDL. Likewise, for the network flux measurements, the IN elements are masked out first.

\section{Results}

\subsection{THE APPEARANCE AND DYNAMICS OF IN FIELDS}

The IN magnetic elements emerge mainly in clusters of mixed polarities from a localized area within a network cell. We call such an area a flux emergence center. In Figure 2, an example of a cluster emergence is shown. The circle drawn at 17:43 UT indicates the site of an emergence center. By 19:50 UT, most of the IN elements in this group had emerged. Although the seeing deteriorated after 16:49 UT, the elements were still plainly visible. Note that this emergence center, unlike most cases, is not located in the center of the network cell. In another such example, an emergence center is right below a network magnetic element. IN elements are seen to move continuously out from the boundary of the network element. Later the network element itself is broken into two fragments as a result.

A typical sized IN element is indicated by an arrow at 16:49 UT in Figure 2. Even though it is visible on the first generation print, the readers may not be able to make it out on theirs. This negative polarity flux measures $-6 \times 10^{16} \mathrm{Mx}$ with an area of $5(\operatorname{arc~sec})^{2}$ and a peak flux density of $-4.8 \mathrm{G}$. Other features of interest in the figure are as follows: IN elements 'canceling' with network of opposite 


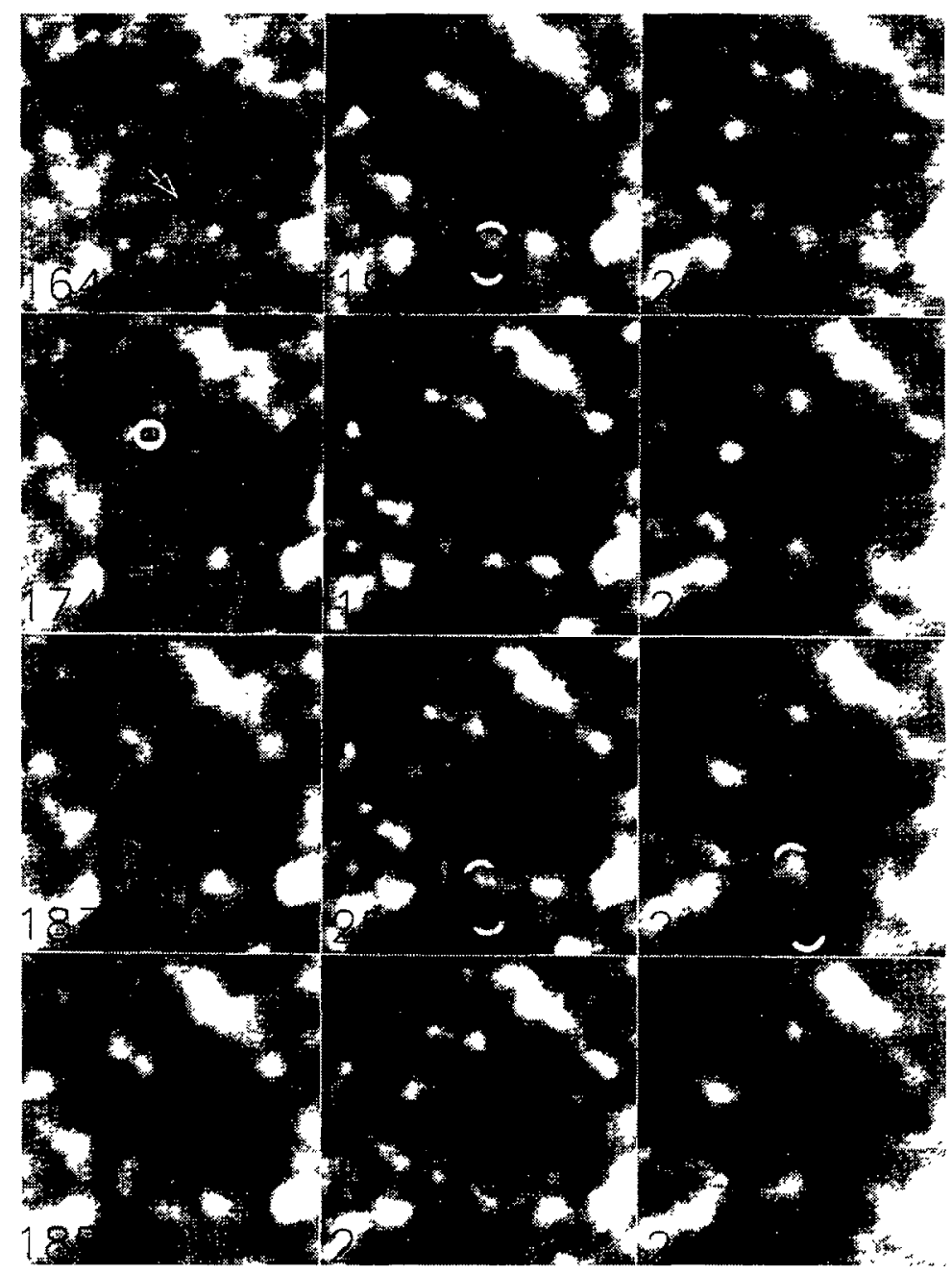

Fig. 2. Time sequence of magnetograms showing the site of an IN emergence center where a cluster of mixed polarity elements emerged (circle); an IN element with flux of $6 \times 10^{16} \mathrm{Mx}$ (arrow at 16:49 UT); an IN element 'canceling' with network of the opposite polarity (a bracket); and a fast moving IN element with an average speed of $0.38 \mathrm{~km} \mathrm{~s}^{-1}$ (to the left of the arrow at 18:55 UT).

polarity (marked with a bracket), and a large positive IN element clearly visible throughout the observation with an average speed of $0.38 \mathrm{~km} \mathrm{~s}^{-1}$. The arrow in the frame of 18:55 UT marks the direction of the motion of the element, which is to the left of the arrow. Later in the 22:13 UT frame, this IN element can be seen merged with a network element of the same polarity. Occasionally a small dipole appears in the cluster. Unlike other IN dipoles that travel in the same general direction, this one grows as the two polarities move in opposite directions, much like an ephemeral region (ER). At maximum development, the magnetic flux of this dipole is $2.5 \times 10^{18} \mathrm{Mx}$, typical for network magnetic elements. Parentheses in Figure 2 indicate the evolution of the small ER. In the seven hours of observation, a total of 16 ephemeral regions are observed, of which 12 have magnetic flux similar to the one shown. 
TABLE I

Statistics of magnetic elements

\begin{tabular}{lll}
\hline & Intranetwork & Network \\
\hline Numbers & 2520 & 536 \\
Total flux $\left(10^{2} \mathrm{Mx}\right)$ & 6.3 & 21.9 \\
Mean flux $\left(10^{17} \mathrm{Mx}\right)$ & $2.5_{-1.5}^{+43}$ & $40.9_{-21.8}^{+60.4}$ \\
Mean area $\left.(\operatorname{arc~sec})^{2}\right)$ & $10.9 \pm 7.6$ & $17.9 \pm 7.4$ \\
Mean $B_{\max }(\mathrm{G})$ & $5.9 \pm 3.0$ & $87.3 \pm 57.7$ \\
\hline
\end{tabular}

TABLE II

Flux distributions in both polarities

\begin{tabular}{llllll}
\hline & \multicolumn{2}{l}{ Intranetwork } & & \multicolumn{2}{l}{ Network } \\
\cline { 2 - 3 } \cline { 5 - 6 } \cline { 5 - 6 } & Positive & Negative & & Positive & Negative \\
\hline Numbers & 1378 & 1142 & & 353 & 183 \\
Total flux $\left(10^{20} \mathrm{Mx}\right)$ & 3.7 & -2.6 & & 15.8 & -6.1 \\
Mean flux $\left(10^{17} \mathrm{Mx}\right)$ & $2.7_{-1.6}^{+45}$ & $-2.3_{-3.8}^{+1.3}$ & & $44.8_{-23.2}^{+53.7}$ & $-33.3_{-73.0}^{+17.7}$ \\
\hline
\end{tabular}

\subsection{STATISTICS}

In the magnetogram shown in Figure 1, 2520 IN magnetic elements and 536 network elements are identified and measured. The statistics are listed in Table I.

The uneven deviations following the means in the table are due to calculations done separately from either side, lower and higher than the means, respectively, and reflect the asymmetrical nature of the distribution profiles. Table II compares the IN flux distribution with that of network elements for both polarities.

Based on the statistics we obtained the following results:

\section{(I) Number Density}

The number densities for the magnetic elements from our results are: 0.74 per $100(\operatorname{arcsec})^{2}$ for network, and 3.50 for IN.

The number density for network bright points identified from the Ca II filtergrams with a spatial resolution of $0.3^{\prime \prime}$ is 0.3 per $100(\operatorname{arc~sec})^{2}$ (Muller, 1983), and 0.6 per $100(\operatorname{arc~sec})^{2}$ from even higher-resolution data (Mehltretter, 1974).

\section{(II) Polarity Balance in IN Fields}

Although the enhanced network fields show predominantly positive flux in our field of view, the intranetwork flux is basically balanced in both polarities to within $70 \%$. This is in agreement with the result of Wang et al. (1989). Taking into account the 
TABLE III

Numbers of IN elements and areas (in square arc sec)

\begin{tabular}{lcccc}
\hline Region & Upper-right & Upper-left & Lower-right & Lower-left \\
\hline IN areas & 15160 & 15250 & 17500 & 16960 \\
IN numbers & 506 & 438 & 883 & 693 \\
Density & 0.03 & 0.28 & 0.05 & 0.04 \\
\hline
\end{tabular}

possibility of misidentifying smaller network elements, which are mostly positive, as IN elements when making the measurements, the balance of the IN flux between two opposite polarities might be even better than that shown in Table II.

\section{(III) Uniform Spatial Distribution}

The region studied can be roughly divided into four parts: the upper right - dominant in enhanced positive network; the upper left - enhanced mixed polarity network; the lower right enhanced negative network; and the lower left - quiet network. However, the numbers of IN flux elements in each of these segments are roughly proportional to the area (total area excluding that occupied by the network elements), independent of the makeup of the network flux. The numbers of IN magnetic elements and areas (in square arc sec) in each of four segments are listed in Table III.

\subsection{FLUX DISTRIBUTIONS}

Flux distributions for IN and network flux are plotted in Figure 3 as asterisks and dots, respectively. The bin size for IN data points is $10^{16} \mathrm{Mx}$, and $10^{18} \mathrm{Mx}$ for network, such that the flux range for either covers about 2 orders of magnitude. The distributions show distinct peaks at $6 \times 10^{16} \mathrm{Mx}$ and $2 \times 10^{18} \mathrm{Mx}$ for IN and network flux, respectively. The number of elements with flux larger than that of the peak basically follows a power law, with power index of -1.68 and -1.27 for IN and network elements, respectively.

\section{Discussion}

\subsection{RELATIVE IMPORTANCE}

The IN magnetic elements are different from network elements in their appearance, emergence, and statistical properties. Although they are small in size and flux, and weak in field strength, their global distribution and rapid life cycle render them potentially important in solar magnetism and atmospheric heating (Parker, 1991). 




Fig. 3. Flux distributions for IN (asterisks) and network flux elements (dots).

Firstly, the IN magnetic elements contribute an average flux density of $1.65 \mathrm{G}$ on the Sun at any given time, which corresponds to a total flux of $10^{23} \mathrm{Mx}$ on the Sun. Since the lifetime of IN fields is much less than a day, about $10^{24} \mathrm{Mx}$ flux on the Sun will emerge and disappear in a single day in the form of IN elements. This is close to the total flux on the whole Sun during the maximum phase of solar cycle 21 (Rabin et al., 1991).

Secondly, unlike what was thought previously, our data show that some IN magnetic elements are actually fairly stable for a few hours (see the IN elements marked at 18:32 and 18:55 in Figure 2). IN elements disappear when they encounter opposite-polarity elements of network, IN, or ephemeral regions. Otherwise they merge with elements of the same polarity, losing their separate identity there. The 'canceling' sites of IN elements with strong network fields of opposite polarity appear to be the main sites of $\mathrm{H} \alpha$ jets and other magnetic activity on the quiet Sun. This will be discussed in a separate paper.

\subsection{THE DOMINANT FLUX SIZE}

The fact that there is a peak in the flux distribution for the network elements (Figure 3 ) is certain since almost all network elements can be identified and measured 
without ambiguity. However, the certainty of a peak in the flux distribution for IN elements needs to be examined with respect to seeing as well as selection effects.

If a magnetograph had an infinite sensitivity, the total flux measured for a unipolar region would remain conserved no matter how poor the seeing is. In reality, the sensitivity is, of course, limited, with a detection threshold, $B_{t}$. Moreover, when the seeing is poor, two nearby elements of opposite polarities may seem to cancel, resulting in an underestimation of the true flux.

To understand the seeing and selection effects, we assume a magnetic element well separated from others, with a gaussian flux distribution and a standard deviation $a$. The point spread function is assumed to be gaussian with a standard deviation $b$, then the total flux one could observe will be

$$
\Phi_{\mathrm{obs}}=\Phi_{\text {true }}\left(1-\frac{a^{2}+b^{2}}{r a^{2}}\right),
$$

where $r$ is the ratio of the true field strength, $B_{r}$, to the threshold flux density, $B_{t}$. It then follows that when

$$
a^{2} B_{r}<\left(a^{2}+b^{2}\right) B_{t},
$$

no flux can be detected for a given element, a case described by Tarbell, Title, and Schoolman (1979) during their search for IN fields. The flux detection limit then should be $\left(a^{2}+b^{2}\right) B_{t}$. This indicates that to detect weak IN lields, not only is a high spatial resolution required, but also a high sensitivity of the magnetograph.

To test if the observed flux distribution is due to a convolution of a power law with seeing and selection effects, we made two simulations, one degenerated by seeing effects, the other by a combination of seeing and selection effects. In the first case we assume $a=100 \mathrm{~km}, b=725 \mathrm{~km}, B_{r}=100 \mathrm{G}$, and $B_{t}=2 \mathrm{G}$ in Equations (1) and (2) for a seeing-degenerated simulation. In the second simulation, we further assume a measurement error such that the possibility of an IN element selected for the correct flux bin is only $30 \%$, with a $70 \%$ possibility of scattering to the nearby three bins on either side. The simulations are based on the observed power-law index of -1.68 . Figure 4 shows the simulations as well as the powerlaw distribution and the observed results. Neither the seeing nor the seeing-plusselection effects reproduce the observed result for this quiet region distribution from a power-law distribution. This renders validity to the observed result.

To examine the results in Figure 3, we take another magnetogram at 20:02 UT when the seeing had deteriorated. The same measurements were made. The new flux distribution is almost the same as that in Figure 3 for network elements; for IN elements it is also very similar, but the peak shifts slightly to a higher flux of $7 \times 10^{16} \mathrm{Mx}$ (from $6 \times 10^{16} \mathrm{Mx}$ ). This agreement further convinces us of the validity of the peak in the flux distribution measured from the best magnetogram.

At present it is difficult to know if a magnetic element represents a single or several elementary flux tubes because observations with sub-arc sec resolution 




Fig. 4. Comparison of pure power law, simulated, and observed flux distributions of IN elements.

are not available. But the fact that there is a dominant flux distribution may shed some light on the physical process that generates and possibly confines the $500 \mathrm{G}$ IN magnetic elements. As network flux concentration is related to supergranular motion, it is natural to ask if IN flux concentration is related to meso-granulation or granulation, the convective cells of smaller scales. Schüssler (1984) deduced that a whirl motion in the downdraft conjunction of granules might stabilize a small flux tube with flux less than $(5-10) \times 10^{17} \mathrm{Mx}$, which is close to the flux range of IN elements.

\subsection{FLUX SOURCE}

If the network fields are all $\mathrm{kG}$ in nature, then the IN elements may represent a different kind of magnetic field that is unrelated to network fields. The results of our study show that they have a distinct peak, a balanced polarity and a spatial uniformity in the flux distribution. We speculate that the IN fields may belong to a different species. Since network fields may be viewed as part of the active region flux, they may be the products of the global solar dynamo; whereas the IN flux, as a different species, may be generated differently as well. 
The small dynamo models proposed by Petrovay and Szakaly (1993) and Durney, Young, and Roxburgh (1993) could be a possible source of IN flux with the following scenario: they are generated by a small-scale dynamo in layers which are not much deeper than the base of supergranules. The subsurface converging motion of supergranules gathers them to the supergranule center, and brings them up to the solar surface. Due to either their small diameter, or weak field strength, they do not experience buoyancy and tension forces, but mainly follow the dynamical drag force of the supergranule motion. Once in the photosphere, if they do not reconnect with other IN magnetic flux elements, they may simply be swept to the network boundary and then either dragged down once again to the base of supergranules to start yet another life cycle, or reconnect with the strong network fields there. If the IN flux tubes are closed small-scale loops, then reconnection or merging would not add or remove much flux, but would merely temporarily break the original topology of magnetic lines of force and cause turbulence in the network fields. The IN fields could not extend to the higher atmosphere, but the network fields could. Thus, the reconnection between network and IN fields may cause turbulence to propagate along the network fields to the upper atmosphere.

In a few cases, we find that IN magnetic elements appear as small ephemeral regions, growing and separating with a maximum flux of $2 \times 10^{18} \mathrm{Mx}$. In these cases, magnetic buoyancy forces may play a dominant role.

\section{Acknowledgements}

J. Wang's work is supported by the collaboration program funded by the National Natural Science Foundation of China and the NSF of the United States. H. Wang, F. Tang, and H. Zirin are supported by NSF Grant INT-9206863 and ONR Grant N00014-89-J-1069. The work was done when J. Wang was visiting Caltech. He wishes to express his gratitude to Prof. Harold Zirin for his warm hospitality. The authors wish to thank the referee, Dr. C. Keller, for his helpful critique.

\section{References}

Durney, B. R., De Young, D. S., and Roxburgh, I. W.: 1993, Solar Phys. 145, 207.

Keller, C. U., Deubner, F. L., Egger, U., Fleck, B., and Povel, H. P.: 1994, Astron. Astrophys. 286, 626.

Lin, H.: 1994, Astrophys. J., submitted.

Liu, Y., Zhang, H., Ai, G., Wang, H., and Zirin, H.: 1994, Astron. Astrophys. 283, 215.

Livi, S. H. B., Wang, J., Martin, S.F.: 1985, Australian J. Phys. 38, 855.

Livingston, W. C. and Harvey, J. W.: 1975, Bull. Amer. Astron. Soc. 7, 346.

Martin, S. F.: 1984, in S. L. Keils (ed.), Small-scale Dynamical Processes in Quiet Stellar Atmospheres, p. 30, Sunspot, NM.

Martin, S. F.: 1988, Solar Phys. 117, 243.

Martin, S. F.: 1990, in J. O. Stenflo (ed.), Solar Photosphere: Structure, Convention, and Magnetic Fields, p. 129. 
Muller, R.: 1983, Solar Phys. 85, 113.

Parker, E. N.: 1991, Astrophys. J. 372, 719.

Petrovay, K. and Szakaly, G.: 1993, Astron. Astrophys. 274, 543.

Rabin, D. M., DeVore, C. R., Sheeley, N. R., Jr., Harvey, K. L., and Hoeksema, J. T.: 1991, in A. N. Cox et al. (eds.), Solar Interior and Atmosphere, p. 781.

Schüssler. M.: 1984, Astron. Astrophys. 140, 453.

Shi, Z., Wang, J., Liu, J., Han, F., and Liu, G.: 1990, Acta Astron. Sinica 31, 63.

Smithson, R. C.: 1975, Bull. Amer. Astron. Soc 7, 346.

Stenflo, J. O.: 1973, Solar Phys. 32, 41.

Tarbell, T. D., Title, A. M., and Schoolman, S. A.: 1979, Astrophys. J. 229, 387.

Varsik, J. R.: 1994, Solar Phys., submitted.

Wang, H. and Zirin, H.: 1988, Solar Phys. 115, 205.

Wang, H., Zirin, H., Patterson, A., Ai, G., and Zhang, H.: 1989, Astrophys. J. 343, 489.

Wang, J. and Shi, Z.: 1988, Acta Astron. Sinica 29, 48.

Wang, J., Zirin, H., and Shi, Z.: 1985, Solar Phys. 98, 241.

Wang, J., Shi, Z., Liu, J., Han, F., and Liu, G.: 1989, Publ. Beijing Astron. Obs. 14.

Zirin, H.: 1985, Australian J. Phys. 38, 961.

Zirin, H.: 1987, Solar Phys. 110, 101.

Zirin, H.: 1993, in H.Zirin et al. (eds.), Magnetic and Velocity Fields of Solar Active Regions, p. 215. 